\title{
Ciencias en PISA 2015: Inmigración, Ansiedad y Apoyo Parental
}

\author{
PISA 2015 Science: Immigration, Anxiety and Support Emotional Parents
}

\author{
Esperanza Bausela Herreras ${ }^{1}$
}

\begin{abstract}
Resumen
Introducción. Los estudios apuntan a diferencias en el rendimiento en la competencia científica en función de diversas variables. Objetivo. Conocer qué variable es mejor predictor del rendimiento en la competencia científica en PISA 2015: condición de inmigrante del estudiante, ansiedad ante los exámenes y el aprendizaje o apoyo parental. Método. No experimental o ex post facto. Instrumento de recogida de datos. Cuestionario PISA 2005. Análisis de datos. Análisis de segmentación (análisis de árboles). Resultados. El porcentaje más alto de estudiantes con bajo rendimiento en Ciencias son estudiantes inmigrantes de primera generación. Discusión y conclusiones. Plantear la importancia de una atención individualizada y personalizada en los planes de acogida para estudiantes inmigrantes. En este proceso de adaptación, también, desempeña un papel fundamental el bienestar psicológico y la percepción de apoyo parental por parte del propio estudiante.
\end{abstract}

Palabras clave: apoyo parental, ansiedad, inmigración, rendimiento

\begin{abstract}
Introduction. Studies point to differences in performance in terms of scientific competence as a function of a range of variables. Objective. To discover which variable best predicts performance in scientific competence in PISA 2015: the student being an immigrant, pre-exam anxiety or parental learning or support. Method. Non-experimental or ex-post facto. Data collection instrument. PISA 2005 survey. Data analysis. Tree segmentation. Results. The greatest percentage of students who perform badly in Science are first-generation immigrants. Discussion and conclusions. Pose the importance of personalised, individual attention as part of induction plans for immigrant students. Psychological well-being and the student's perception of parental support also play key roles in this adaptation process.
\end{abstract}

Keywords: support emotional parents, anxiety, immigration, performance

\footnotetext{
${ }^{1}$ Dk. Área de Psicología Evolutiva y de la Educación - Hezkuntza eta Garapen Psikologiaren Alorra. Campus de Arrosadia s/n. Universidad Pública de Navarra | Nafarroako Unibertsitate Publikoa. Correo: esperanza.bausela@unavarra.es 


\section{Introducción}

Las diferencias en el rendimiento en las evaluaciones internacionales es una realidad (ver Gil, Martínez, \& Sáez, 2019). PISA (Programme for International Student Asesment) es un estudio que es desarrollado trianualmente por la OCEDE (Organización para la Cooperacion y el Desarrollo Económico) que evalúa a estudiantes de 15 años en diversas competencias. En la edición de 2015 el foco de atención se puso en la competencia científica. Los resultados obtenidos en estos estudios condicionan no sólo las políticas educativas, sino que también nos permiten conocer qué variables inciden en el rendimiento con un doble objetivo, bien minimizar su impacto o bien para maximizarlas, desarrollando propuestas psicopedagógicas acordes a las mismas.

En el presente estudio nos vamos a centrar, por su relevancia, en tres varaibles: Condición de inmigrante (IMMIG), Ansiedad relativa a los exámenes y el aprendizaje (ANXTEST) y Percepción de los estudiantes del apoyo emocional parental (EMOSUPS).

\section{Condición inmigrante del estudiante}

Los movimientos migratorios han cambiado la composición de los centros educativos y de las clases (Brunello, \& Rocco, 2011).

Las evidencias empíricas apuntan a que los escolares inmigrantes obtienen puntuaciones más bajas que sus compañeros nativos (Dustamnn, Frattinn, \& Theodorpoulos, 2011).

A pesar, de la abundante investigación en relación a la misma (Dustmann, Frattini, \& Lanzara, 2011; Murat, \& Frederic, 2011) y de su importancia, se sabe relativamente poco sobre qué factores pueden predecir el rendimiento académico de los estudiantes inmigrantes y los factores que permiten explicar las diferencias en su rendimiento al compararlo con sus compañeros nativos (Böhlmark, 2008; Brunello, \& Rocco, 2013; Calero, \& Escardíbul, 2013; CebollaBoado, 2011; Chowa, Masa, Wretman, \& Ansong, 2013; de Lange, Dronkers, \& Wolbers, 2013; Geay, McNally, \& Telhaj, 2013; Gould, Lavy, \& Paserman, 2009; Hampden, 2013; Hillmert, 2013; Winsler, 2014; Ohinata, \& van Ours, 2013).
Son diversas las variables que puede estar incidiendo, como por ejemplo, la concentración de los propios estudiantes inmigrantes en un centro (Cebolla-Boado, 2011, 2012; Jensen \& Rasmussen, 2011) ya que no es posible generar el conflicto socio cognitivo defendido por Vygotsky (1979) que contribuye al aprendizaje por la propio homogeneidad del centro. Otra variable que incide en el rendimiento es la etapa de llegada del estudiante inmigrante (Bausela, 2017; Glick, Hanish, Yabiku, \& Bradley, 2012; Stiefl, Schwartz, \& Conger, 2010; Van Ours \& Veenman, 2006).

Los inmigrantes tienen que hacer frente a diversos y diferentes desafíos cuando se incorporan al Sistema Educativo del país de acogida (Organización para la Cooperación y el Desarrollo Económicos (OCDE, 2015, Motti et al., 2008; Torres \& Rollock, 2004): deben adaptarse rápidamente a diferentes expectativas académicas, aprender una nueva lengua que es el vehículo que les va a facilitar el acceso al currículo, forjar una identidad social que facilite su adaptación, todo ello, en ocasiones, bajo presiones contradictorias de la propia familia, existiendo variaciones en función de la generación a la que pertenecen y de los patrones de afrontamiento preferidos entre los estudiantes migrantes.

\section{Ansiedad}

Diferentes estudios enfatizan los altos niveles de estrés que experimentan los escolares inmigrantes en este proceso de adaptación a la nueva realidad (Arbona et al., 2010; García \& Lindgren, 2009; Torres \& Rollock, 2004). Las investigaciones apuntan hacia una asociación entre la condición de inmigrante y el estrés psicológico (Hatzenbuehlera et al, 2017). Achotegui (2009), concretamente, hace referencia al síndrome del inmigrante con estrés crónico y múltiple o síndrome de Ulises. Este síndrome es conocido como un trastorno a medio camino entre los trastornos adaptativos y el trastorno por estrés postraumático.

La propia OCDE (2018) en el informe The Resilience of Students with an Immigrant Background señala: "Pero asegurar que los estudiantes de origen inmigrante tienen bienestar representa un reto significativo, porque muchos de ellos deben sobreponerse a las adversidades relacionadas con el desplazamiento, la desventaja 
socioeconómica, las barreras de la lengua y la dificultad de buscar una nueva identidad al mismo tiempo".

Los problemas de ansiedad pueden incidir de forma negativa en el rendimiento académico, abandono escolar e inhabilidad general para alcanzar las metas trazadas (Center for Addiction and Mental Health, 2012). Serrano, Rojas y Ruggero (2012) señalan una asociación entre el nivel de ansiedad y el rendimiento en estudiantes inmigrantes.

En los propios estudios de PISA 2015 los resultados indican que los escolares inmigrantes de primera generación presentan mayor ansiedad relacionada con la elaboración de los deberes escolares, existiendo una diferencia de 6\% respecto a sus compañeros nativos.

Son diversos los estudios que incluyen esta variable o variables próximas a la misma en sus estudios para conocer como incide en las personas inmigrantes. Murillo y Molero (2012) analizan, por su parte, tres factores que inciden en el bienestar de la población migrante y otros grupos minoritarios: prejuicio, identidad grupal y bienestar económico. La relación entre los factores económicos y el bienestar subjetivo no es lineal. Así, Palomar, Montes de Ocaia, Polo y Estrada (2016) analizan variables escolares, familiares y sociales y su relación con el rendimiento académico de escolares inmigrantes mexicanos que residen en Nueva York. Orozco (2013) incide en factores y características internas de los inmigrantes, factores socioculturales de la sociedad receptora, contexto económicosociocultural, factores sociodemográficos (edad, educación y género), factores geográficos, adaptación sociocultural, aculturación, estrés aculturativo y adaptación psicológica, recursos y estrategias de superación. Torres y Rollock (2004) hacen referencia a los patrones de afrontamiento, al proceso de aculturación y a la adaptación psicológica.

\section{Apoyo parental}

La participación de los padres y el apoyo escolar de estudiantes inmigrantes es un aspecto esencial, así Ruiz de Miguel ${ }^{1}$ y Castro, Expósito, López, Lizasoain, Navarro y Gaviria (2015) lo

${ }^{1}$ http://blog.intef.es/inee/2015/03/12/la-implicacion-de-los-padres-y-elapoyo-escolar-claves-para-el-exito-de-alumnos-inmigrantes/ concluyen en sus estudios. Los resultados de los estudios constatan una relación bidireccional entre las pautas de crianza y el bienestar emocional (Davidov \& Grusec, 2006). Yotyodying y Wild (2014), por su parte, señalan la existencia de diferencias en relación a la instrucción parental en el hogar dependiendo de la cultura (Alemania versus Tailandia). Freund, Schaedel, Azaiza, Boehm y Lazarowitz (2018), también encuentran diferencias culturales, concluyendo que los padres árabes muestran una mayor participación en la escuela y la comunidad que los padres judíos.

Castro et al. (2015) encuentran una asociación alta entre hábitos lectores y las familias que tienen altas expectativas académicas para sus hijos que desarrollan y mantienen comunicación con ellos sobre las actividades escolares. Los hallazgos de Dumont et al. (2012) resaltan la necesidad de conceptualizaciones diferenciadas de la participación de los padres, así como análisis detallados de los procesos subyacentes a la asociación entre antecedentes familiares y resultados educativos.

Los estudios son contundentes sobre la implicación, participación y apoyo parental de los padres de estudiantes inmigrantes en el proceso de enseñanza - aprendizaje de sus hijos (Aparicio, 2003; Bueno y Belda, 2005; Fernández et al., 2002; González, 2007).

En este contexto nos planteamos como objetivo conocer qué variable es mejor predictor del rendimiento en la competencia científica evaluada en PISA 2015: Condición de inmigrante (IMMIG), Ansiedad relativa a los exámenes y el aprendizaje (ANXTEST) y Percepción de los estudiantes del apoyo emocional parental (EMOSUPS).

\section{Método}

Metodología cuantitativa. No experimental, en el contexto de los estudios ex -post-facto, basado en la aplicación del cuestionario PISA 2015 2, .

\footnotetext{
${ }^{2}$ OECD (versión original) <http://www.oecd.org/pisa/pisaenespaol.htm > (fecha de consulta: 20 de abril de 2017).

${ }^{3}$ Instituto Nacional de Evaluación Educativa (INEE) de España (versión traducida y adaptada al español)

$<$ https://www.educacionyfp.gob.es/inee/evaluaciones-

internacionales/pisa/pisa-2015.html> (fecha de consulta: 20 de abril de 2017).
} 
Tabla 1. Pregunta de PISA 2015 "Ansiedad relativa a los exámenes y el aprendizaje"

¿En qué medida estás en desacuerdo o de acuerdo con las siguientes afirmaciones sobre ti mismo? (Por favor, selecciona una respuesta en cada fila)

\begin{tabular}{lcc}
\hline Preguntas & $\begin{array}{c}\text { Muy en } \\
\text { desacuerdo }\end{array}$ & $\begin{array}{c}\text { En } \\
\text { desacuerdo }\end{array}$ acuerdo \\
\hline
\end{tabular}

Con frecuencia me preocupa que el examen me resulte difícil.

(ST118Q01NA)

Me preocupa sacar malas notas en clase. (ST118Q02NA)

Incluso cuando estoy bien preparado para un examen me encuentro

muy nervioso. (ST118Q03NA)

Me pongo muy tenso cuando estudio para un examen.

(ST118Q04NA)

Me pongo nervioso cuando no sé resolver un ejercicio en clase.

(ST118Q05NA)

Fuente: Ministerio de Educación, Cultura y Deporte (2017).

\section{Participantes}

En este estudio se ha utilizado la base de datos de PISA 2015 extraída de la página de la $\mathrm{OCDE}^{4}$ y disponible en la página institucional de Instituto Nacional de Evaluación Educativa (INEE) de España $^{3}$. La muestra está constituida por 39066 jóvenes españoles que se distribuyen en: 6736 (17.2\%) son estudiantes que no se adscriben a ninguna región y 32330 (82.8\%) pertenecen a alguna de las CC.AA. La técnica de muestreo utilizada es bietápico por conglomerados.

\section{Instrumento de recogida de datos}

Cuestionario PISA 2015,6 que evalúa los conocimientos y destrezas en las áreas de Lectura, Matemáticas y Ciencias. En la edición de 2015, el estudio PISA se centró en en la competencia científica, dejando las competencias en lectura, matemáticas y resolución colaborativa de problemas como áreas secundarias de la evaluación (OECD, 2017).

\section{Variables de investigación}

En relación al papel que desempeñan en la investigación las variables son:

i) Variable dependiente (endógena): Rendimiento en competencia científica

Esta competencia es definida como la habilidad para interactuar con cuestiones relacionadas con la ciencia y con las ideas de la ciencia, como un

\footnotetext{
${ }^{4}$ OECD <http://www.oecd.org/pisa/data/2015database/> (fecha de consulta: 20 de abril de 2017).

${ }^{5}$ OECD (versión original)

<http://www.oecd.org/pisa/pisaenespaol.htm> (fecha de consulta: 20 de abril de 2017).

${ }^{6}$ INEE (versión traducida y adaptada al español)

$<$ https://www.educacionyfp.gob.es/inee/evaluaciones-

internacionales/pisa/pisa-2015.html> (fecha de consulta: 20 de abril

de 2017).
}

ciudadano reflexivo.

Se ha utilizado el valor plausible 1 para ciencias disponible en la base de datos de PISA 2015. En PISA 2015 se han utilizado 10 valores plausibles.

ii) Variables independientes o predictoras (exógena):

\section{Condición de inmigrante (IMMIG)}

Esta variable se incluye en los estudios PISA dentro de los "Factores demográficos de los estudiantes para evaluar la igualdad y la equidad" junto con otras variables: género, discapacidad, nivel socioeconómico y pobreza, idioma hablado en el hogar e idioma de instrucción.

En PISA 2015 la condición de inmigrante se basa en un enfoque basado en preguntas sobre el lugar de nacimiento de los jóvenes o estudiantes y sus padres. Se define como estudiantes inmigrantes aquéllos que no han nacido en el país donde se aplica la prueba y que además tienen un padre o madre no nacidos en dicho país (Ministerio de Educación, Cultura y Deporte, 2016).

\section{Ansiedad relativa a los exámenes y el aprendizaje (ANXTEST)}

Esta variable se incluye en los estudios PISA 2015 dentro de los factores de salud y bienestar que a su vez pertenece al módulo de "Resultados de Prosperidad" junto con otras variables: desempeño académico, logros educativos e involucración estudiantil.

Ansiedad, es un constructo complejo que en PISA 2015 se ha centrado en las reacciones cognitivas y emocionales hacia el trabajo escolar, especialmente el estudio y los exámenes (Ministerio de Educación, Cultura y Deporte, 2017). 
Tabla 2. Pregunta de PISA 2015 "Percepción de los estudiantes del apoyo emocional parental"

En relación con este curso, ¿en qué medida estás de acuerdo o en desacuerdo con las siguientes afirmaciones? (Por favor, selecciona una respuesta en cada fila)

\begin{tabular}{|c|c|c|c|c|}
\hline Preguntas & $\begin{array}{c}\text { Muy en } \\
\text { desacuerdo }\end{array}$ & $\begin{array}{c}\text { En } \\
\text { desacuerdo }\end{array}$ & $\begin{array}{c}\text { De } \\
\text { acuerdo }\end{array}$ & $\begin{array}{l}\text { Muy de } \\
\text { acuerdo }\end{array}$ \\
\hline
\end{tabular}

Mis padres se interesan por mis actividades escolares. (ST123Q01NA)

Mis padres apoyan mis esfuerzos y logros educativos. (ST123Q02NA)

Mis padres me apoyan cuando me enfrento a dificultades escolares. (ST123Q03NA)

Mis padres me animan para que tenga confianza en mí mismo.

(ST123Q04NA)

Fuente: Ministerio de Educación, Cultura y Deporte (2017).

Tabla 3. Distribución de los participantes en función de las variables independientes estudiadas

\begin{tabular}{lcc}
\hline Variables independientes & Frecuencia & Porcentaje \\
\hline Condición del estudiante & & \\
Nativo & 34083 & 87.2 \\
Secunda Generación & 661 & 1.7 \\
Primera generación & 3473 & 8.9 \\
No Responde/No válido & 849 & 2.2 \\
Ansiedad relativa a los exámenes y el aprendizaje (ANXTEST) & & \\
Alto (1.5126_.6572) & 14013 & 35.9 \\
Medio (.6571_.0753) & 10415 & 26.7 \\
Bajo (.0572_-.6871) & 14147 & 36.2 \\
No Responde/No válido & 491 & 1.3 \\
Percepción de los estudiantes del apoyo emocional parental (EMOSUPS) & & \\
Alto (.5659_.1005) & 20242 & 51.8 \\
Medio (.1004_-.4262) & 6730 & 17.2 \\
Bajo (-.4263_-1.3869) & 11613 & 29.7 \\
No Responde/No válido & 481 & 1.2 \\
\hline
\end{tabular}

Fuente: Elaboración propia a partir de PISA 2015 (muestra española).

Las preguntas formuladas a los estudiantes se presenta en la Tabla 1.

Las respuestas de los estudiantes se utilizaron para construir un índice de ansiedad (ANXTEST), relacionado con el trabajo escolar, que se ha estandarizado de forma que tiene media 0 y desviación típica 1 en el conjunto de países de la OCDE (Ministerio de Educación, Cultura y Deporte, 2017).

\section{Percepción de los estudiantes del apoyo emocional parental (EMOSUPS)}

Esta variable se incluye en los estudios PISA al igual que la anterior dentro de los factores de "Salud y bienestar" pertenece al módulo "Resultados de Prosperidad".

PISA recopila datos de los estudiantes sobre su percepción del apoyo parental y de los padres sobre las actividades que hacen con sus hijos o en los centros de sus hijos (Ministerio de Educación, Cultura y Deporte, 2017). La pregunta formulada a los estudiantes se presenta en la Tabla 2.
Las respuestas de los estudiantes se utilizaron, al igual que con la anterior variable, para construir un índice de percepción de los hijos del apoyo emocional parental (EMOSUPS) que se ha estandarizado de forma que tiene media $0 \mathrm{y}$ desviación típica 1 en el conjunto de países de la OCDE (Ministerio de Educación, Cultura y Deporte, 2017).

En la Tabla 3 se presenta la distribución de los participantes en las tres variables independientes analizadas: Condición de inmigrante (IMMIG), Ansiedad relativa a los exámenes y el aprendizaje (ANXTEST) y Percepción de los estudiantes del apoyo emocional parental (EMOSUPS):

i) 4134 escolares son inmigrantes representan el $10.6 \%$ de los participantes, que han ido llegado desde los 0 meses de vida hasta los 16 años de vida. De los cuales el $1.7 \%$ pertenecen a segundas generaciones (661 estudiantes) y el $8.9 \%$ pertenecen a la primera generación (3473 estudiantes). 
Tabla 4. Resumen del modelo de segmentación

\begin{tabular}{llc}
\hline & Método de crecimiento & CHAID \\
& Variable dependiente & Valor Plausible 1 para Ciencias \\
Especificaciones & Variables independientes & Index Immigration status, ANSTEST_B_M_A, \\
& Validación & noSUP_B_M_A \\
& Máxima profundidad del árbol & 3 \\
& Casos mínimos en nodo padre & 100 \\
& Casos mínimos en nodo hijo & 50 \\
\hline \multirow{2}{*}{ Resultados } & Variables independientes incluidas & Index Immigration status, ANSTEST_B_M_A, \\
& Número de nodos & EMOSUP_B_M_A \\
& Número de nodos terminales & 20 \\
& Profundidad & 13 \\
\hline
\end{tabular}

Fuente: Elaboración propia a partir de PISA 2015 (muestra española).

ii) En relación a Ansiedad relativa a los exámenes y el aprendizaje (ANXTEST): 14013 (35.9\%) de los estudiantes presentan alto nivel y un porcentaje ligeramente superior de estudiantes 14147 (36.2\%) presenta bajo nivel.

iii) Finalmente, respecto a la percepción de los estudiantes del apoyo emocional parental (EMOSUPS): 20242 estudiantes, más de la mitad de los participantes (51.8\%) tienen una alta percepción de apoyo por parte de sus padres mientras que 11613 estudiantes (29.7\%) tienen baja percepción del mismo.

\section{Análisis de datos}

Técnica de análisis de segmentación que según Escobar (2007) es una técnica de dependencia entre variables que ha sido aplicada con el mismo objetivo en otros estudios (Bravo, Pavez, Salvo, \& Mieres, 2019; Bravo, Salvo, Mieres, Mansilla, \& Hederich, 2017; Bravo, Salvo, \& Muñoz, 2015). En su aplicación se distinguen: (i) Variables cuya distribución se desea a explicar (variable dependiente, competencia científica) y (ii) Variables, nominales $\mathrm{u}$ ordinales, con estatus independientes que reciben el nombre de predictores y tienen por finalidad formar grupos que sean muy distintos entre sí en la variable o variables dependientes: Condición de inmigrante (IMMIG), Ansiedad relativa a los exámenes y el aprendizaje (ANXTEST) y Percepción de los estudiantes del apoyo emocional parental (EMOSUPS).

Se ha utilizado el SPPS versión 24 (módulo de clasificar "árbol") para manejar la base de datos de PISA 2015.

\section{Resultados}

Se ha procedido a realizar un análisis de segmentación con el objeto de descubrir qué qué variables predicen el rendimiento de los estudianteslos estudiantes en la competencia científica en PISA 2015.

Se ha utilizado la técnica CHAID de segmentación, que utiliza el criterio básico de no permitir segmentaciones que no sean estadísticamente significativas. Los límites de significación se sitúan en el nivel 0.05 que se corresponde con un nivel de confianza del 95\%. Las características del modelo se resumen en la Tabla 4.

El árbol de decisión (Figura 1) nos muestra:

- En primer lugar, nos fijamos en el nodo 0 que describe la variable dependiente, la media en el rendimiento en la competencia científica de toda la muestra es 499.111.

- Seguidamente observamos que la variable dependiente se ramifica en cuatro nodos: Nodo 1 (Nativos), Nodo 2 (Primera Generación), Nodo 3 (Segunda generación) y Nodo 4 (perdidos) pertenecientes a la variable predictora "Condición de Inmigrante". El Nodo 1 nos indica que los escolares nativos obtienen una media superior en la competencia científica en comparación con los escolares inmigrantes de primera y segunda generación y en los casos que no se ha identificado esta condición.

- El Nodo 1 (escolares nativos) se ramifica en 11 Nodos en función de la variable predictora "Ansiedad relativa a los exámenes y el aprendizaje". Los nodos obtenidos nos muestran que los estudiantes con niveles medio-bajo de ansiedad (Nodo 5 y Nodo 7) obtienen 

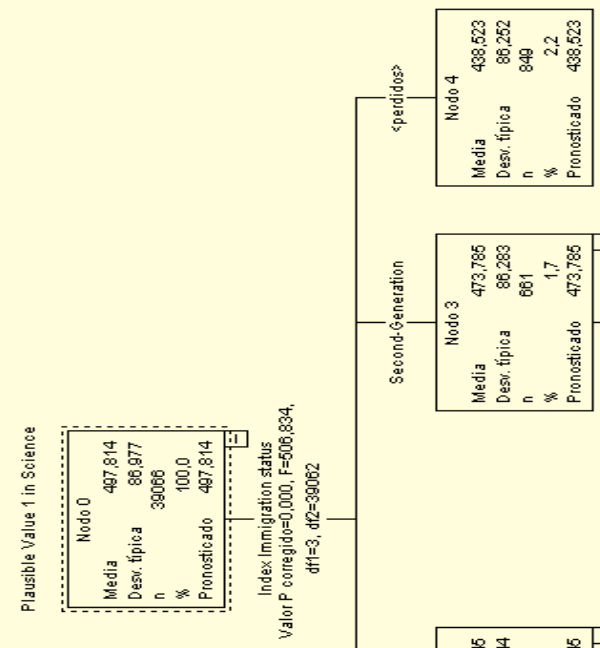

$\frac{n}{2}$
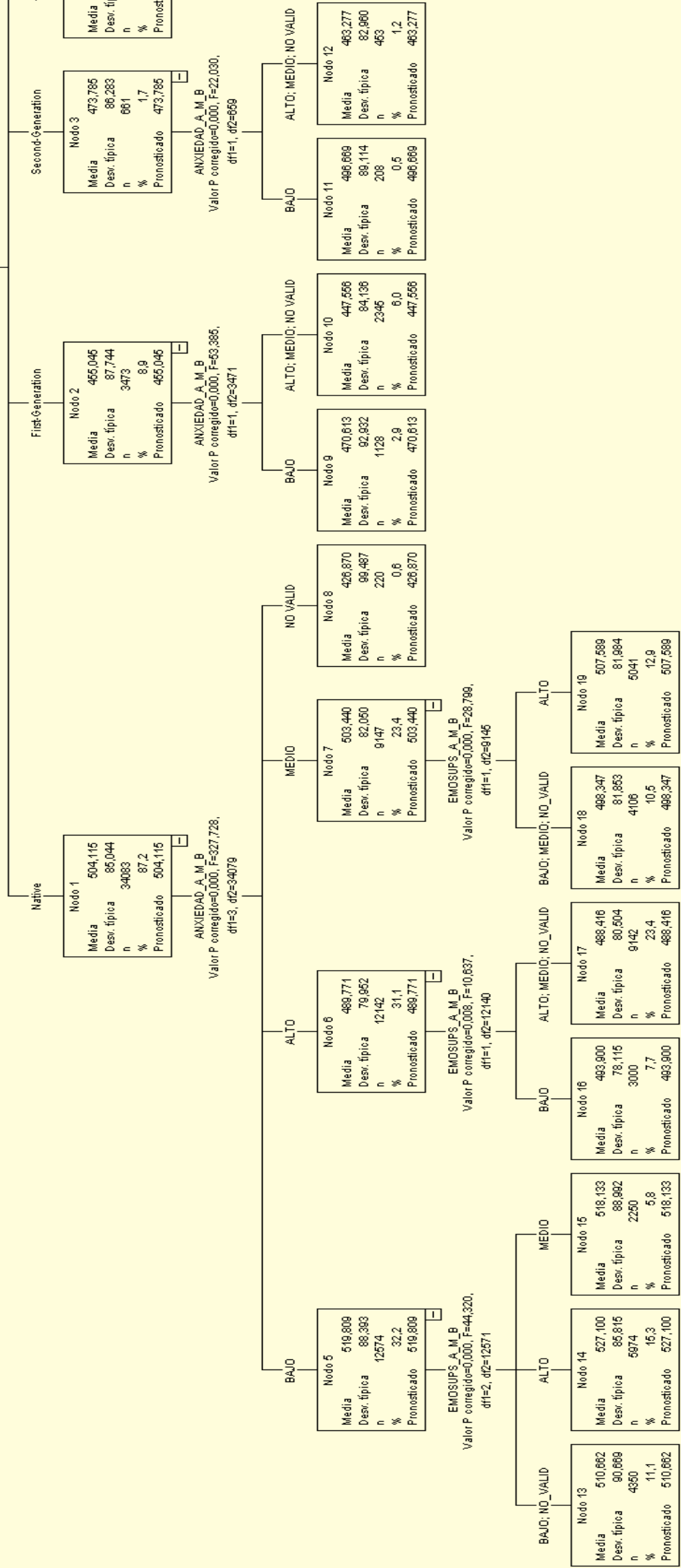
puntuaciones más altas en la competencia científica en comparación con los resultados que obtienen los estudiantes con niveles más altos en ansiedad (Nodo 6).

- Los nodos con estudiantes nativos y niveles medios de ansiedad (Nodo 7) Se ramifican en dos nodos cada uno de ellos en función de la variable predictora "Percepción de los estudiantes del apoyo emocional parental". Así, el se divide el nodo 7 en el Nodo 18 y Nodo 19. En este nivel se observa que ha igual nivel de ansiedad obtiene una media más alta en competencia científica los estudiantes que tienen mayor percepción de apoyo emocional parental (Nodo 19).

En la Tabla 5 podemos observar las ganancias para los nodos, el cual nos ofrece un resumen de información sobre los nodos terminales del modelo. Con frecuencia, los nodos terminales despiertan interés ya que representan los mejores pronósticos de clasificación para el modelo.

Podemos observar que el nodo 17 tienen un índice teniendo mayor probabilidad de frecuencia ( $n=9142)$ caracterizándose por ser: estudiantes nativos; con alto nivel de ansiedad relativa a los exámenes y el aprendizaje; alto-medio nivel de percepción de apoyo emocional parental; y un rendimiento en competencia científica media de 488.41569 .

Tabla 5. Resumen de ganancias para nodos

\begin{tabular}{lccc}
\hline Nodo & $\mathrm{N}$ & Porcentaje & Media \\
\hline 14 & 5974 & $15.3 \%$ & 527.10026 \\
15 & 2250 & $5.8 \%$ & 518.13301 \\
13 & 4350 & $11.1 \%$ & 510.66241 \\
19 & 5041 & $12.9 \%$ & 507.58902 \\
18 & 4106 & $10.5 \%$ & 498.34684 \\
11 & 208 & $0.5 \%$ & 496.66949 \\
16 & 3000 & $7.7 \%$ & 493.90020 \\
17 & 9142 & $23.4 \%$ & 488.41569 \\
9 & 1128 & $2.9 \%$ & 470.61290 \\
12 & 453 & $1.2 \%$ & 463.27726 \\
10 & 2345 & $6.0 \%$ & 447.55586 \\
4 & 849 & $2.2 \%$ & 438.52252 \\
8 & 220 & $0.6 \%$ & 426.87008 \\
\hline
\end{tabular}

Fuente: Elaboración propia a partir de PISA 2015 (muestra española). Método de crecimiento: CHAID. Variables dependiente: Valor Plausible 1 para Ciencias

La estimación del riesgo es de 7065,675 mal clasificados con una desviación del error de 47,846 en relación al total de participantes 38853 que representado el $18.8 \%$ de los participantes (Tabla 6).

\begin{tabular}{lcc}
\multicolumn{3}{c}{ Tabla 6. Riesgo } \\
\hline $\begin{array}{l}\text { Estimación } \\
\text { Plausible Value 1 in }\end{array}$ & Desv. Error \\
$\begin{array}{l}\text { Science } \\
\text { Fuente: Elaboración propia a partir de PISA } 2015\end{array}$ \\
$\begin{array}{l}\text { española). Método de crecimiento: } \\
\text { dependiente: Valor Plausible 1 in Science }\end{array}$
\end{tabular}

\section{Discusión}

Este estudio tenía como objetivo conocer qué variables eran las mejores predictoras del rendimiento académico en Ciencias en los estudios PISA 2015, considerando el rendimiento académico como una variable continua y utilizando el primer valor palusible para Ciencias disponible en PISA 2015. Las variables analizadas han sido: Condición de inmigrante (IMMIG), Ansiedad relativa a los exámenes y el aprendizaje (ANXTEST) y Percepción de los estudiantes del apoyo emocional parental (EMOSUPS).

Los resultados obtenidos tras desarrollar una técnica de segmentación (árbol de segmentación) nos indica que el modelo discrimina entre variables sociales y variables vinculadas con el bienestar de los estudiantes. Así, en los tres niveles de rendimiento los nodos iniciales se corresponden con la "Condición de inmigrante". En el segundo nivel de los árboles se corresponde con la variable "Ansiedad relativa a los exámenes y el aprendizaje”. En el tercer nivel se sitúan los nodos relacionados con la "Percepción de los estudiantes del apoyo emocional parental" para estudiantes nativos, en el caso de los estudiantes de estudiantes "inmigrantes de primera y segunda generación" no se llega hasta este nivel de profundidad por la baja frecuencia de estudiantes con este nivel.

Cabe destacar en relación a la "Condición de inmigrante" que el porcentaje más alto de estudiantes con bajo rendimiento en Ciencias son estudiantes inmigrantes de primera generación, mientras que el rendimiento medio y alto rendimiento se asocia con estudiantes nativos.

Estos datos nos muestran que los estudiantes inmigrantes son quienes tienen un riesgo mayor de tener bajo rendimiento en la competencia científica en comparación con sus compañeros estudiantes nativos. Se confirman los resultados obtenidos por diferentes investigadores (Böhlmark, 2008; Brunello \& Rocco, 2013; 
Calero \& Escardíbul, 2013; Cebolla-Boado, 2011; Chowa, Masa, Wretman, \& Ansong, 2013; de Lange, Dronkers, \& Wolbers, 2013; Geay, McNally, \& Telhaj, 2013; Gould, Lavy, \& Paserman, 2009; Hampden, 2013; Hillmert, 2013; Winsler, 2014; Ohinata \& van Ours, 2013).

Un segundo aspecto a desatacar es el papel que tiene la "Ansiedad relativa a los exámenes y el aprendizaje". Los resultados nos muestran como la ansiedad es una variable que incrementa el riesgo de tener bajo rendimiento en esta competencia científica. Estos resultados se encuentran en la misma dirección que los obtenidos por Arbona et al. (2010), García y Lindgren (2009), Torres y Rollock (2004) y Vallejo y Zuleta (2019).

Finalmente, en relación a la "Percepción de los estudiantes del apoyo emocional parental", los resultados se adhieren a los obtenidos por otros investigadores que destacan el papel que tiene la familia en el rendimiento (de Lange, Dronkers \& Wolbers, 2013; Hampden, 2013; Reparaz \& Sotés, 2018).

Estas evidencias nos llevan a plantear la importancia de una atención individualizada y personalizada en los planes de acogida para estudiantes inmigrantes, estudiantes de incorporación tardía o estudiantes con especiales condiciones en su historia vital en las primeras etapas del Sistema Educativo (Bausela, 2017). Por otro lado, es necesario ser sensible a necesidades relacionadas con el bienestar psicológico (Moutinho, Monteiro, Costa, \& Faria, 2019) y no sólo a necesidades educativas y/o lingüístico, que repercutirá de forma positiva en todos los estudiantes en general (OECD, 2011).

En definitiva, la llegada a nuestro Sistema Educativo de estudiantes inmigrantes debe suponer un reto y un desafío para el mismo contribuyendo a su desarrollo (Rahona, \& Morales, 2013) y una oportunidad de cambio y avance. Cuanto más comprehensivo sea un Sistema Educativo con la diversidad es de esperar que sea más fácil la adaptación del estudiante. En este proceso de adaptación, también, desempeña un papel fundamental para todos los escolares el bienestar psicológico y la percepción de apoyo parental por parte del propio estudiante.

En este estudio no se pueden establecer relaciones de causalidad entre las variables analizadas, siendo necesario profundizar en estudios que permitan conocer la relación entre dichas variables y el rendimiento en las tres competencias evaluadas en PISA 2015. Se ha considerado una única dimensión del bienestar emocional, siendo necesario considerar una visión multidimensional de éste constructo que incluye aspectos relacionados con los ámbitos: académico, social, emocional y motivación. Somos conscientes de que tres variables no pueden contribuir a explicar el rendimiento en la competencia científica, ya que no llegan a explicar el $1 \%$ de la variabilidad promedio del rendimiento, pero estamos atravesando una situación de crisis sanitaria derivada del COVI-19 que nos obliga a centrarnos en tres variables que pueden contribuir a explicar una brecha en el rendimiento: ansiedad a los exámenes y al aprendizaje (por la incertidumbre, por el cambio radical de una metodología docente presencial a on line a distancia); percepción de los estudiantes del apoyo parental (conciliación familia-escuela, con incertidumbre laboral, con actividades académicas sustituyendo a los docentes) y estatus inmigrante (que pueden verse aún más acentuadas las diferencias entre estudiantes nativos e inmigrantes por la utilización de recursos informáticos condicionados por el status socioeconómico de los participantes). Son tres variables, as que hemos analizado en este estudio, pero estimamos que probablemente en estos momentos las propuestas y políticas educativas no deben olvidarlas ni obviarlas e incidir para que este momento no conlleve más desventajas y acentúe las diferencias entre los estudiantes en situaciones de desventaja social.

\section{Conflicto de interés}

La autora manifiesta que no tiene conflicto de intereses.

\section{Referencias}

Achotegui, J. (2009). Estrés límite y salud mental: El síndrome del inmigrante con estrés crónico y múltiple (síndrome de Ulises). Gaceta Médica de Bilbao, 106(4), 122-133. doi:10.1016/S0304-4858(09)74665-7 
Aparicio, R. (2003). El entorno familiar de los menores de origen extranjero escolarizados en Madrid. Madrid: Universidad Pontificia de Comillas y Ministerio de Migraciones y Servicios Sociales.

Arbona, C., Olvera, N., Rodríguez, N., Hagan, J., Linares, A., \& Wiesner, M. (2010). Acculturative stress among documented and undocumented Latino immigrants in the United States. Hispanic Journal of Behavioral Sciences, 32(3), 362-384. doi:10.1177/0739986310373210

Bausela, E. (2017). PISA 2012: Inmigración y etapa de llegada al Sistema Educativo Español. Revista Costarricense de Psicología, $36(1), 1-22$.

Berlanga, V., Rubio, M. J., \& Vilà, R. (2013). Cómo aplicar árboles de decisión en SPSS. Reire Revista d'innovació $i$ Recerca en Educació, 6(1), 65-79.

Böhlmark, A. (2008). Age at immigration and school performance: A sibling's analysis using Swedish register data. Labour Economics, 15(6), 1366-1387. doi: 10.1016/j.labeco.2007.12.004.

Bravo, M., Pavez, M., Salvo, S., \& Mieres, M. (2019). Autoeficacia, expectativas y violencia escolar como mediadores del aprendizaje en Matemática. Revista Espacios, 40(33), 28.

Bravo, M., Salvo, S., \& Muñoz, C. (2015). Profiles of Chilean students according to academic performance in mathematics: An exploratory study using classification trees and random forests. Studies in Educational Evaluation, 44, 50-59. doi:10.1016/j.stueduc.2015.01.002

Bravo, M., Salvo, S., Mieres, M., Mansilla, J., \& Hederich, C. (2017). Perfiles de desempeo académico: La importancia de las expectativas familiares. Perfiles Latinoamericanos, 25(50), 361-386. doi:10.18504/pl2550-016-2017

Bronfenbrenner, U. (1976). The ecology of human development: History and perspectives. Psychologia, 19 (5), 537-549.

Brunello, G., \& Rocco, L. (2013). The effect of immigration on the school performance of natives: Cross country evidence using PISA test scores, Economics of Education Review, 32, 234-246. doi:10.1016/j.econedurev.2012.10.006
Brunello, G., \& Rocco, L. (2011). The effect of immigrant ton the school performance of native: Cross country evidence using PISA test scores. IZA Discussion Papers Series, 5479.

Bueno, J. R., \& Belda, J. F. (Dirs.) (2005). Familias inmigrantes en la escuela. Discursos de los agentes educativos. Valencia: Universidad de Valencia.

Calero, J., \& Escardíbul, O. (2013). El rendimiento del alumnado de origen inmigrante en PISA- 2012, En MEC y OCDE (Ed.), PISA 2012: Programa para la evaluación internacional de los alumnos. Informe español. Volumen II: Análisis secundario (pp. 4-31). Madrid: MEC y OCDE.

Castro, M., Expósito, E., López, E., Lizasoain, L., Navarro, E., \& Gaviria, J. L. (2015). Parental involvement on student academic achievement: A meta-analysis. Educational Research Review, 14, 33-46. doi:10.1016/j.edurev.2015.01.002

Cebolla-Boado, H. (2011). Primary and secondary effects in the explanation of immigrants' educational disadvantage. British Journal of Sociology, 32, 407-430. doi:10.1080/01425692.2011.559341.

Cebolla-Boado, H. (2012). La incorporación escolar de la población de origen inmigrante y el impacto de la concentración de inmigrantes en las escuelas navarras, Navarra: Departamento de Políticas Sociales. Sección de Atención a la Inmigración. Gobierno de Navarra.

Centre for Addiction and Mental Health (2012). Anxiety disorders. <http://www.camh.ca/en/ hospital/health_information/a_z_mental_healt h_and_addiction_information/anxiety_disorde rs/Pages/Anxiety_Disorders.asp>

Chowa, G. A. N., Masa, R.D., Wretman, C. J., \& Ansong, D. (2013). The impact of household possessions on youth's academic achievement in the Ghana Youth save experiment: A propensity score analysis. Economics of Education Review, 33, 69-81. doi:10.1016/j.econedurev.2012.08.005

Davidov, M., \& Grusec, J. E. (2006). Untangling the links of parental responsiveness to 
distress and warmth to child outcomes. Child Development, 77, 44-58. doi:10.1111/j.1467-8624.2006.00855.x

de Lange, M., Dronkers, J., \& Wolbers, M.H.J. (2013). Single - parent family forms and children's educational performance in a comparative perspective: Effects of school's share of single-parent families. School Effectiveness and School Improvement, 1-22. doi:10.1080/09243453.2013.809773

Dumont, H., Trautwein, U., Lüdtke, O., Neumann, M., Niggli, A., \& Schnyder, I. (2012). Does parental homework involvement mediate the relationship between family background and educational outcomes? Contemporary Educational Psychology, 37(1), 55-69. doi:10.1016/j.cedpsych.2011.09.004

Dustmann, C., Frattini, T., \& Lanzara, G. (2011). Educational achievement of second generation immigrants: An international comparison. Centro Studi Luca D'Agliano Development Studies WP, 314.

Dustmann, C., Frattini, T., \& Theodorpoulos, N. (2011). Ethnicity and second generation immigrants. In (P. Gregg and J. Wadsworth, eds.), The labour market in winter: The state of working Britain (pp. 220-39). Oxford: Oxford University Press.

Entforf, H., \& Minoiu, N. (2005). What a difference inmigration policy makes: A comparison of PISA scores in Europe and traditional countries of inmigration. German Economic Review, 6(3), 355-376. doi:10.1111/j.1468-0475.2005.00137.x

Escobar, M. (2007). El análisis de segmentación: Técnicas y aplicaciones de los árboles de clasificación. Madrid: Centro de Investigaciones Sociológicas.

Fernández Sierra, J., et al. (2002). Estudio de las actitudes, creencias y actuaciones educativas del profesorado andaluz ante el alumnado inmigrante. Consejería de Gobernación. Junta de Andalucía. Proyecto de Investigación. Curso 2001/02.

Freund, A., Schaedel, B., Azaiza, F., Boehm, A., \& Lazarowitz, R. H. (2018). Parental involvement among Jewish and Arab parents: Patterns and contextual predictors. Children and Youth Services Review, 85, 194-201. doi:10.1016/j.childyouth.2017.12.018
Garcia, C., \& Lindgren, S. (2009). "Life grows between the rocks": Latino adolescents' and parents' perspectives on mental health stressors. Research in Nursing \& Health, 32(2), 148-62. doi:10.1002/nur.20317

Geay, C., McNally, S., \& Telhaj, S. (2013). Nonnative speakers of English in the classroom: What are the effects on pupil performance? The Economic Journal, 123, F281-F307. doi:10.1111/ecoj.12054

Gil, P., Martínez, M., \& Sáez, M.B. (2019). Objective and subjective factors associated with Spanish students' performance in science in PISA 2015 / Factores objetivos y subjetivos asociados al rendimiento del alumnado español en ciencias en PISA 2015. Culture and Education, 31(4), 671-715. doi:10.1080/11356405.2019.1656485

Glick, J. E., Hanish, L. D., Yabiku, S. T., \& Bradley, R. (2012). Migration timing and parenting practices: Contributions to social development in preschoolers with foreignborn and native-born mothers. Child Development, 83(5), 1527-1542. doi:10.1111/j.1467-8624.2012.01789.x

Gonzalo, I. (2007). La participación de las familias inmigrantes en la escuela: Necesidades de orientación y formación. XXI. Revista de Educación, 9, 155-169.

Gould, E. D., Lavy, V., \& Paserman, M.D. (2009). Does immigration affect the long term educational outcomes of natives? Quasi experimental evidence. The Economic Journal, 119, 1243-1269. doi:10.1111/j.1468-0297.2009.02271.x.

Hampden, G. (2013). Family policy, family structure, and children's educational achievement, Social Science Research, 42(3), 804-817. doi:10.1016/j.ssresearch.2013.01.005

Hatzenbuehlera, M. L., Prins, S. J., Flake, M., Philbin, M., Somjen, M., Hagen, D., \& Hirsch, J. (2017). Immigration policies and mental health morbidity among Latinos: A state-level analysis. Social Science \& Medicine, 174, 169-178. doi:10.1016/j.socscimed.2016.11.040

Hillmert, S. (2013). Links between immigration and social inequality in education: $\mathrm{A}$ comparison among five European countries, 
Research in Social Stratification and Mobility, 32, 723. doi:10.1016/j.rssm.2013.02.002

Jensen, P., \& Rasmussen, A. (2011). The effect of immigrant concentration in schools on native and immigrant children's reading and math skills. Economics of Education Review, 30(6), 1503-1515.

doi:10.1016/j.econedurev.2011.08.002

López, E., Expósito, E., Molina, E. C., \& Muñoz, I. A. (2018). What does PISA tell us about the teaching and learning of sciences? An approach through decision trees. Revista de Educacion, 382, 133-161. doi:10.4438/1988-592X-RE-2018-382-395

Ministerio de Educación, Cultura y Deporte (MECD) (2016). PISA 2015. Programa para la evaluación internacional de los alumnos. Informe Español (versión preliminar). Madrid: Autor.

Ministerio de Educación, Cultura y Deporte (MECD) (2017). PISA 2015. Programa para la Evaluación Internacional de los Alumnos. El Bienestar de los estudiantes. Informe español. Madrid: Autor.

Motti, F., Pavlopoulos, V., Obradovi'c, J., Dalla, M., Takis, N., \& Papapthanassiou, A. (2008). Immigration as a risk factor for adolescent adaptation in Greek urban schools. European Journal of Developmental Psychology, 5(2), 235-261. doi:10.1080/17405620701556417

Moutinho, H., Monteiro, A., Costa, A., \& Faria, L. (2019). Papel da inteligência emocional, felicidade e flow no desempenho académico e bem-estar subjetivo em contexto universitário. Revista Iberoamericana de Diagnóstico y Evaluación - e Avaliação Psicológica, 52(3), 99-114. doi:10.21865/ridep52.3.08

Murat, M., \& Frederic, P. (2011). Institutions, culture and background: The school perfomance of inmigrant students. Education Economics, 31, 612-630. doi:10.1080/09645292.2014.894497

Murillo, J., \& Molero, F. (2012). Factores psicosociales asociados al bienestar de inmigrantes de origen colombiano en España. Psychosocial Intervention, 21(3), 319-329.

Ohinata, A, \& van Ours, J. C. (2013). How inmigrant children affect the academic achievement of native dutch children. The Economic Journal, 103, F308-F331. doi:10.1111/ecoj.12052

Organización para la Cooperación y el Desarrollo Económicos (OECD) (2011). Cómo se están adaptando los sistemas escolares al creciente número de estudiantes inmigrantes. PISA IN FOCUS, 11. OECD Publishing: Paris.

Organización para la Cooperación y el Desarrollo Económicos (OECD) (2015). Can the performance gap between immigrant and non - immigrant students be closed? PISA IN FOCUS, 7. OECD Publishing: Paris.

Organización para la Cooperación y el Desarrollo Económicos (OCDE) (2017). Marco de Evaluación y de Análisis de PISA para el Desarrollo: Lectura, matemáticas y ciencias. Versión preliminar. OECD Publishing: Paris

Organización para la Cooperación y el Desarrollo Económicos (OECD) (2018). The Resilience of Students with an Immigrant Background. OECD Publishing: Paris.

Orozco, V. A. E. (2013). Migración y estrés aculturativo: Una perspectiva teórica sobre aspectos psicológicos y sociales presentes en los migrantes latinos en Estados Unidos. Norteamérica, 8(1), 7-44. doi:10.1016/S1870-3550(13)71765-8.

Palomar, J., Montes de Oca, S. I., Polo, A. M., \& Estrada, A. V. (2016). Factores explicativos del rendimiento académico en hijos de inmigrantes mexicanos en Nueva York. Psicologia Educativa, 22(2), 125-133. doi:10.1016/j.pse.2016.03.001

Rahona, M., \& Morales, S. (2013). Educación e inmigración en España: Desafíos y oportunidades. Series reformas educativas. Madrid: OEI con el apoyo de la Fundación Alternativas.

Reparaz, C., \& Sotés, M. A. (2018). Parental involvement in schools in Spain and Germany: Evidence from PISA 2015. International Journal of Educational Research, 93, 33-52. doi:10.1016/j.ijer.2018.10.001

Serrano, C., Rojas, A., \& Ruggero, C. (2013). Depresión, ansiedad y rendimiento académico en estudiantes universitarios. Revista Intercontinental de Psicología y Educación, 15(1), 47-60.

Stiefl, L., Schwartz, A.E., \& Conger, D. (2010). Age of entry and the high school performance 
of immigrant youth. Journal of Urban Economics, 67, 303-314. doi:10.1016/j.jue.2009.10.001.

Torres, L., \& Rollock, D. (2004). Acculturative distress among hispanics: The role of acculturation, coping, and intercultural competence. Journal of Multicultural Counseling and Development, 32(3), 155-167. doi:10.1002/j.2161-1912.2004.tb00368.x

Vallejo, A., \& Zuleta, K. (2019). Depresión, ansiedad y actividad física en escolares: Estudio Comparado. Revista Iberoamericana de Diagnóstico y Evaluación - e Avaliação Psicológica, 52(3), 143-155. doi:10.21865/ridep52.3.11

Van Ours, J. C., \& Veenman, J. (2006). Age at immigration and educational attainment of young immigrants. Economics Letters, 90, 310-316. doi:10.1016/j.econlet.2005.08.013.

Vygotsky, L. S. (1979). El desarrollo de los procesos psicológicos superiores. Buenos Aires: Grijalbo.

Winsler, A. (2014). Early development among dual language learners: The roles of language use at home, maternal immigration, country of origin, and socio-demographic variables. Early Childhood Research Quarterly, 29(4), 750-764. doi:10.1016/j.ecresq.2014.02.008

Yotyodying, S., \& Wild, E. (2014). Antecedents of different qualities of home-based parental involvement: Findings from a cross-cultural study in Germany and Thailand. Learning, Culture and Social Interaction, 3(2), 98-110. doi:10.1016/j.lcsi.2014.02.002 